\title{
Liquid Chromatographic Determination of Malvidin-3-O-Glucoside and Malvidin 3, 5-O-Diglucoside in Wine Samples by Direct Injection
}

\author{
A. Rodríguez-Bernaldo de Quirós, J. López-Hernández* and M.A. Lage-Yusty
}

\author{
Analytical Chemistry, Nutrition and Bromatology Department, Pharmacy Faculty, Campus Sur s/n, University of \\ Santiago de Compostela, 15782 Santiago de Compostela (La Coruña), Spain
}

\begin{abstract}
A method for the determination of malvidin 3-O-glucoside (Oenin), and malvidin 3, 5- $O$-diglucoside (Malvin) in wines by on line HPLC coupled with UV, and fluorescence detectors is described. With the proposed method the samples were analysed by direct injection without a previous treatment.

For method validation, satisfactory recoveries $(>95 \%)$ and suitable repeatabilities (within day: R.S.D. $(\mathrm{n}=6=)<3 \%$, and between days; R.S.D. $(\mathrm{n}=6=)<3 \%)$ were achieved.
\end{abstract}

The method was applied to the analysis of commercially available red wines.

Keywords: Chromatographic analysis, direct injection, malvin, oenin, wine.

\section{INTRODUCTION}

Malvidin 3-O-glucoside (Oenin) and malvidin 3, 5- $O$ diglucoside (Malvin) are some of the anthocyanins present in wines. In Vitis vinifera species, only anthocyanidinmonoglucosides are present [1,2]; among these, oenin is the predominant anthocyanin in red wines. Malvidin 3, 5- $\mathrm{O}$ diglucoside has been found in hybrid varieties [1]. The individual anthocyanin composition is characteristic of each grape cultivar, so that, the anthocyanin profile has recently been used as a suitable tool to classify wines [3-6].

These compounds are responsible for organoleptic characteristics such as colour, bitterness, and astringency [7-10]. Since the colour is often a criterion of quality in foodstuffs, the analysis of these pigments has received considerable attention in the past years. Furthermore, anthocyanins have also been reported to show anti-inflammatory or antiviral activities, which have been attributed to their antioxidant properties [11].

Numerous methods have been described in the literature for determining anthocyanins in grape and wine samples $[2,3,5,8]$. The most commonly used is liquid chromatography in combination with UV or diode array detectors (DAD) $[1,2,3,5]$ however, the lack of suitable standards makes, in some cases, necessary the identification by mass spectrometry[3]. Recently, liquid chromatography coupled to mass spectrometry has been successfully applied for the characterization of anthocyanins in natural products [7, 12], however this technique is very expensive and is not common in control laboratories. To our knowledge, there are very few studies on the fluorescence properties of these phenolic compounds [13]. The use of a fluorescence detector in series

*Address correspondence to this author at the Analytical Chemistry, Nutrition and Bromatology Department, Pharmacy Faculty, Campus Sur s/n, University of Santiago de Compostela, 15782 Santiago de Compostela (La Coruña), Spain; Tel: +34 981 598450; Fax: +34 981 594912;

E-mail: qnlhjul@usc.es with a UV-Vis detector could be an excellent tool to complement the information provided by the DAD.

In the present paper, we report the optimisation of a high performance liquid chromatographic method with UV-Vis and fluorescence detection to analyse malvidin 3-Oglucoside and malvidin 3, $5 \mathrm{O}$-diglucoside in wine samples. The method was validated in terms of linearity, repeatabilities within day and between days, recoveries and anthocyanins stabilities. LC-FLD has been used as complementary technique. The proposed method could be a useful technique for control and routine analysis.

\section{MATERIALS AND METHODOLOGY}

\subsection{Reagents and Standard Solutions}

All reagents were of analytical grade. Acetonitrile and acetic acid were from Merck (Darmstadt, Germany). Formic acid was obtained from Riedel-de Haën (Seelze, Germany). Malvidin-3-glucoside chloride (Oenin chloride) and malvidin 3,5-diglucoside chloride (Malvin chloride) were supplied by Fluka BioChemika (Steinheim, Germany).

Water used for all solutions was obtained from a Milli-Q water purification system (Millipore) (Bedford, MA, USA).

Standard solutions were prepared in acidified methanol $(0,5 \% \mathrm{v} / \mathrm{v} \mathrm{HCl})$ and stored at $4{ }^{\circ} \mathrm{C}$ in the dark.

\subsection{Samples}

Commercially, available red wines from different parts of Spain were purchased in a local supermarket. The samples were filtered through a $0.50 \mu \mathrm{m}$ PTFE membrane filter (Advantec MFS, INC, CA, USA) and directly injected into the chromatograph. The analyses were performed in triplicate immediately after bottle opening. The rest of the wine was transferred to amber bottles in order to protect against sunlight and was stored in the refrigerator. 


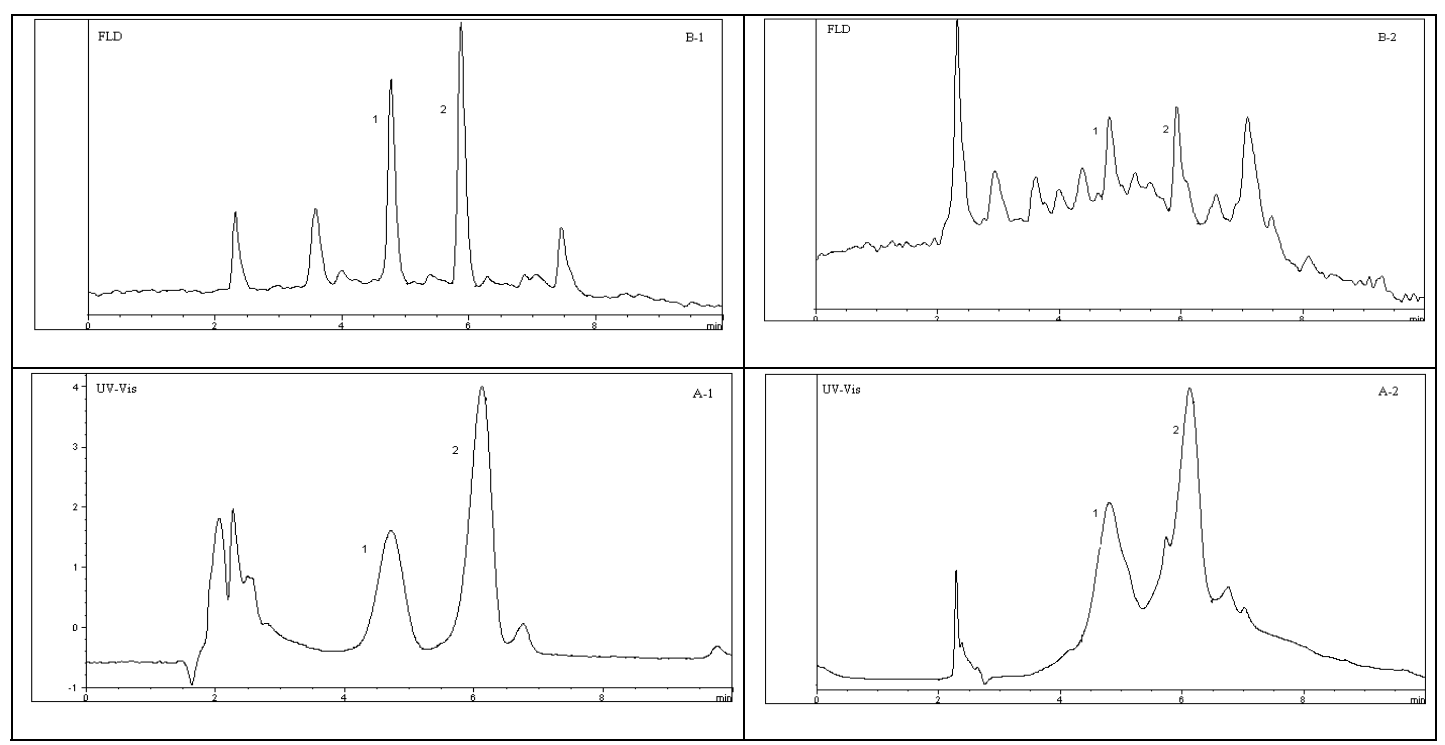

Fig. (1). HPLC-chromatograms of a standard solution and a red wine sample with UV-Vis detector (A-1, A-2), and fluorescence detector (B1,B-2); registered under the optimized chromatographic conditions. Peaks (1) malvin; (2) oenin.

\subsection{Equipment}

Analyses were performed using a Hewlett Packard Series 1100 chromatographic system equipped with a quaternary pump, a degassing device, a $20 \mu \mathrm{L}$ injection loop (Rheodyne, Cotati,California, USA), an UV-Vis detector, and a fluorescence detector. The HPLC system was controlled by a personal computer running (HP) Chem Station Software.

Chromatographic separation was carried-out on a TEKNOKROMA, Tr-015605 TRACER EXTRASIL ODS2 $(25 \mathrm{~cm} \mathrm{X} 0.4 \mathrm{~cm}$ i.d. $5 \mu \mathrm{m})$ controlled at $28^{\circ} \mathrm{C}$ with a column thermostatting system (Spectra Physics 8792, San José, CA, USA).

\subsection{HPLC Analysis}

Operating conditions were as follows: flow rate 1.5 $\mathrm{mL} / \mathrm{min}$, injection volume $20 \mu \mathrm{L}$, and column temperature $28^{\circ} \mathrm{C}$. The mobile phases consisted of (A) water-formic acid -acetic acid (1000:8:9, v/v/v) pH 2 and (B) acetonitrile. The elution conditions applied were: 0-10 min linear gradient from $10-45 \% \mathrm{~B}$, and finally, $100 \% \mathrm{~B}$ for $5 \mathrm{~min}$ before returning to the initial conditions.

A wavelength of $520 \mathrm{~nm}$ was used for UV-Vis detector, and $\lambda_{\text {ex }} 378 \mathrm{~nm}$, and $\lambda_{\text {em }} 435 \mathrm{~nm}$ for fluorescence detector.

\subsection{Identification and Quantification}

The identification of the compounds was made by comparison of their retention times with those of pure standards and by spiking the samples with standard solutions.

Quantitation was performed on the basis of linear calibration plots of peak area against concentration. Calibration lines were constructed based on six concentration levels of standard solutions within $0.5-10 \mathrm{mg} / \mathrm{L}$ range.

\section{RESULTS AND DISCUSSION}

\subsection{Method Optimization}

The chromatographic procedure is a modification of the method proposed by Farina et al. [14]. The gradient elution used to separate anthocyanins is generally based on methanol or acetonitrile-water and an acid modifier, such as acetic acid formic acid, trifluoroacetic acid or perchloric acid. Because of their suitable physico-chemical properties, [3] formic acid is one of the most used chemical. In developing the method, several changes in the proportion of the mobile phase components were assayed; A (water-formic-acid-acetic acid, 1000: 7:9 v/v/v) pH 2.2 B (acetonitrile); A (water-formicacid-acetic acid, 1000:7.5:9 v/v/v) pH $2.1 \mathrm{~B}$ (acetonitrile), and A (water-formic-acid-acetic acid, 1000: 8:9 v/v/v) $\mathrm{pH} 2$; $\mathrm{B}$ (acetonitrile), a better resolution was achieved at $\mathrm{pH} 2$. A low $\mathrm{pH}$ in the mobile phase is essential in order to guarantee that the anthocyanins are in the flavylium form $[3,9]$. Furthermore, at acidic $\mathrm{pH}$, the anthocyanins present the maximum of absorbance at $520 \mathrm{~nm}$, this wavelength is very selective since it is characteristic of these pigments [3]. The effect of the column temperature on the separation was also investigated, the temperature was varied between $20^{\circ} \mathrm{C}$ and $35^{\circ} \mathrm{C}$, and an improvement of the separation was obtained when the column was termostated at $28^{\circ} \mathrm{C}$.

Finally, several flow-rates $(0.8 ; 1 ; 1.2 ;$ and $1.5 \mathrm{~mL} / \mathrm{min})$ were tried, with a suitable separation being achieved with the highest reduction in the analysis time.

\subsection{LC-FLD as a Complementary Technique}

Although the HPLC-UV-Vis is the preferred method for routine analysis of anthocyanins, the use of a fluorescence detector in series with a UV-Vis detector could be an excellent tool to complement the information provided by the UVVis detector. To our knowledge, there are very few reports on fluorescence properties of the anthocyanins [13]. LC-MS is the most widespread method for the identification of these pigments; however, this equipment is very expensive, and is not common in control laboratories.

In spite of the fact that fluorescence response is lower than the signal obtained with UV detector, when a complex sample, like wine, is analysed, the on-line use of the fluorescence detector coupled to the HPLC-UV-Vis system could help us to distinguish between different compounds. 
Table 1. Repeatability of the Method within Day, and Between Days Calculated as the Relative Standard Deviation for Six Replicate Injections at Four Concentrations Levels (\% R.S.D. $(\mathbf{n}=6)$ )

\begin{tabular}{|c|c|c|c|}
\hline \multirow{2}{*}{ Analyte } & \multirow{2}{*}{ Concentration (mg/L) } & \multicolumn{2}{|c|}{ \%RSD (n=6) } \\
\cline { 2 - 4 } & & Within Day & Between Days \\
\hline \hline \multirow{3}{*}{ Malvidin, 3,5-o-diglucoside } & 0.63 & 2 & 2 \\
\cline { 2 - 4 } & 1.25 & 2 & 2 \\
\cline { 2 - 4 } & 2.50 & 2 & 2 \\
\hline \multirow{3}{*}{ Malvidin, 3-o-monoglucoside } & 5.00 & 1 & 3 \\
\cline { 2 - 4 } & 0.63 & 3 & 2 \\
\cline { 2 - 4 } & 1.25 & 1 & 2 \\
\hline
\end{tabular}

The optimal wavelengths for FLD were determined by scanning standards. The best wavelengths were found to be $\lambda_{\text {exc }} 378 \mathrm{~nm}$ and $\lambda_{\mathrm{em}} 435 \mathrm{~nm}$.

Fig. (1) shows chromatograms of a standard solution, and a red wine sample obtained using a UV-Vis and a FLD.

\subsection{Performance Characteristics}

The method was validated with respect to linearity, repeatabilities within day and between days, recoveries and anthocyanins stability in standard solution and in samples.

The linearity of the method was tested by using a series of oenin and malvin standard solutions of known concentration. The calibration curves were constructed using six concentration levels and they were fitted to a linear equation $\mathrm{y}=$ $6.5( \pm 0.06) \mathrm{x}+3( \pm 0.3)$ for malvin and $\mathrm{y}=35( \pm 0.09) \mathrm{x}+0.2$ $( \pm 0.4)$ for oenin; the linear range was within the $0.5-10 \mathrm{mg} / \mathrm{L}$ range. Each point of the calibration curve is the average of three peak-area measurements. Both compounds showed a good linearity. The coefficients of determination obtained were 0.9996 and 0.99996 for malvin and oenin respectively. The limits of detection, calculated according ACS guidelines [15] (defined as signal three times the height of the noise level) were 0.1 for malvin and $0.09 \mathrm{mg} / \mathrm{L}$ for oenin.
The repeatabilities within day and between days were determined by analysing six replicates of standards at four concentration levels $(0.63 ; 1.25 ; 2.5$ and $5 \mathrm{mg} / \mathrm{L})$ on the same day and on three separate days, respectively. The results are presented in Table $\mathbf{1 .}$

Recoveries were estimated on the basis of determination after spiking the samples with known amounts of malvin and oenin standards at three concentration levels. As it can be observed, recoveries higher than $95 \%$ were achieved (Table 2).

Since the method is proposed for control and routine analysis, the stability of the compounds in wine samples was evaluated during $48 \mathrm{~h}$, simulating a thermostatted autosampler $\left(4^{\circ} \mathrm{C}\right)$. No significant degradation was observed. The standard solution prepared in acidified methanol $(0.5 \% \mathrm{v} / \mathrm{v}$ $\mathrm{HCl})$ showed to be stable for ten days at $4^{\circ} \mathrm{C}$ in the dark.

\subsection{Samples Analysed}

Table 3 shows the quantities of oenin and malvin found in seven commercial red wines. The content of oenin ranged from 2.43 to $7.1 \mathrm{mg} / \mathrm{L}$. The differences could be attributed to different factors such as vintage, technology, etc. Malvin was only found in three samples. The quantities detected

Table 2. Recoveries, Determined by Spiking Six Samples of Red Wine with Malvin, and Oenin Standards at Three Concentration Levels (\% \pm S.D. $(n=6))$

\begin{tabular}{|c|c|c|}
\hline \multirow{2}{*}{ Analyte } & \multirow{2}{*}{ Spike (mg/L) } & Recoveries \\
\hline & & $(\operatorname{Mean}(\%) \pm$ S.D. $(n=6)$ \\
\hline \multirow{3}{*}{ Malvin } & 0.8 & $95.3 \pm 1,8$ \\
\hline & 1.6 & $95.2 \pm 3,4$ \\
\hline & 3.6 & $97.3 \pm 1,6$ \\
\hline \multirow{3}{*}{ Oenin } & 0.6 & $95.9 \pm 1,5$ \\
\hline & 1.3 & $97.3 \pm 0,7$ \\
\hline & 3.4 & $97.6 \pm 1,1$ \\
\hline
\end{tabular}


Table 3. Malvin and Oenin Content $( \pm$ SD) $(n=3)$ in Commercial Available Red Wine. Each Sample was Analyzed in Triplicate

\begin{tabular}{|c|c|c|c|c|c|c|c|}
\hline Analyte & Sample 1 & Sample 2 & Sample 3 & Sample 4 & Sample 5 & Sample 6 & Sample 7 \\
\hline \hline Malvin & $\mathrm{Nd}$ & $3.63 \pm 0.36$ & $\mathrm{Nd}$ & $1.09 \pm 0.12$ & $\mathrm{nd}$ & $\mathrm{nd}$ & $5.87 \pm 0.16$ \\
\hline Oenin & $6.46 \pm 0.16$ & $4,17 \pm 0.23$ & $4.24 \pm 0.09$ & $4.08 \pm 0.06$ & $2.43 \pm 0.03$ & $5.79 \pm 0.06$ & $7.1 \pm 0.19$ \\
\hline
\end{tabular}

Nd: not detected.

$\mathrm{N}=3( \pm \mathrm{SD})$.

Conc: $\mathrm{mg} / \mathrm{L}$.

were very low, becoming impossible to conclude if the wines were of hybrid variety [16].

\section{ACKNOWLEDGEMENTS}

A. Rodríguez-Bernaldo de Quirós thanks to Xunta de Galicia for the research grant Parga Pondal. The technical assistance of P. Ferraces-Casais is gratefully acknowledged.

\section{REFERENCES}

[1] Berente B, Reichenbächer M, Danzer K. Improvement of the HPLC analysis of anthocyanins in red wines by use of recently developed columns. Fresenius J Anal Chem 2001; 371: 68-72.

[2] Berente B, De la Calle D, Reichenbächer M, Danzer K. Method development for the determination of anthocyanins in red wines by high-performance liquid chromatography and classification of German red wines by means of multivariate statistical methods. J Chromatogr A 2000; 871: 95-103.

[3] de Villiers A, Vanhoenacker G, Majek P, Sandra P. Determination of anthocyanins in wine by direct injection liquid chromatographydiode array detection-mass spectrometry and classification of wines using discriminant analysis. J Chromatogr A 2004; 1054: 195-204.

[4] Nollet LML Food Analysis by HPLC. $2^{\text {nd }}$ ed. Basel: Marcel Dekker, Inc 2000.

[5] Revilla E, García-Beneytez E, Cabello F, Martín-Ortega G, Ryan JM. Value of high-performance liquid chromatographic analysis of anthocyanins in the differentiation of red grape cultivars and red wines made from them. J Chromatogr A 2001; 915: 53-60.

[6] Gómez-Ariza JL, García-Barrera T, Lorenzo F. Anthocyanins profile as fingerprint of wines using atmospheric pressure photoionization coupled to quadrupole time-of-flight mass spectrometry. Anal Chim Acta 2006; 570: 101-8.

[7] Alcalde Eon C, Saavedra G, de Pascual-Teresa S, Rivas-Gonzalo JC. Liquid chromatography-mass spectrometry identification of an- thocyanins of isla oca (Oxalis tuberosa, Mol.) tubers. J Chromatogr A 2004; 1054: 211-5.

[8] Revilla E, Ryan JM. Analysis of several phenolic compounds with potential antioxidant properties in grape extracts and wines by high-performance liquid chromatography-photodiode array detection without sample preparation. J Chromatogr A 2000; 881: 461469.

[9] da Costa, CT, Horton D, Margolis SA. Analysis of anthocyanins in foods by liquid chromatography, liquid chromatography-mass spectrometry and capillary electrophoresis. J Chromatogr A 2000; 881: 403-10.

[10] Lee HS, Hong V. Chromatographic analysis of anthocyanins. J Chromatogr 1992; 624: 221-34.

[11] Bridle P, Timberlake CF. Anthocyanins as natural food coloursselected aspects. Food Chem 1997; 58: 103-09.

[12] Montoro P, Tuberoso CIG, Perronea A, Piacentea S, Cabrasb P Pizza CJ. Characterisation by liquid chromatography-electrospray tandem mass spectrometry of anthocyanins in extracts of Myrtus communis L. berries used for the preparation of myrtle liqueur. J Chromatogr A 2006; 1112: 232-40.

[13] Drabent R, Pliszka B, Olszewska T. Fluorescence properties of plant anthocyanin pigments. I. Fluorescence of anthocyanins in Brassica oleracea L. extracts. J Photochem Photobiol B Biol 1999; 50: 53-58.

[14] Farina A, Doldo A, Cotichini V, et al. HPTLC and reflectance mode densitometry of anthocyanins in Malva Silvestris L.: a comparison with gradient-elution reversed-phase HPLC. J Pharm Biomed Anal 1995; 14: 203-11.

[15] American Chemical Society (ACS), Subcommittee of Environmental Analytical Chemistry. Guidelines for data acquisition and data quality evaluation in environmental chemistry. Anal Chem 1980; 52: 2242-49.

[16] Gutiérrez-Fernández Y, Rivas-Gonzalo JC, Santos-Buelga C. Comparison of red wines on the basis of mono- and diglucoside anthocyanins. Alimentaria 1992; 29: 27-31. 